\title{
Prediction of Experimental Electromagnetic Coupling to a UAV Model Using Characteristic Mode Analysis
}

\author{
Mohamed Z. M. Hamdalla, Benjamin Bissen, James D. Hunter, Liu Yuanzhuo, Victor Khilkevich, \\ Daryl G. Beetner, Anthony N. Caruso, and Ahmed M. Hassan
}

\begin{abstract}
In this work, we study the current coupled to a simplified Unmanned Aerial Vehicle (UAV) model using a dual computational and experimental approach. The surrogate structure reduced the computational burden and facilitated the experimental measurement of the coupled currents. For a practical system, a wide range of simulations and measurements must be performed to analyze the induced current variations with respect to the incident excitation properties such as the frequency, angle of incidence, and polarization. To simplify this analysis, Characteristic Mode Analysis (CMA) was used to compute the eigen-currents of the UAV model and predict where and under which RF excitation conditions, the coupled current is maximized. We verified these predictions using direct experimental measurement of the coupled currents. The presented simulations and measurements show the usefulness of CMA for studying electromagnetic coupling to practical systems.
\end{abstract}

Index Terms - Field to wire coupling, UAV, Electromagnetic coupling, characteristic mode analysis.

\section{INTRODUCTION}

Unmanned aerial vehicles (UAVs) have a wide range of applications, and typically operate in congested wireless environments that cause unwanted interference [1]-[5]. The commercial UAV industries have recently expressed interest in using UAVs as mobile base stations [6]-[9], and as backhaul infrastructure [6]-[9], which further exacerbate their intra- and inter-induced electromagnetic compatibility (EMC) and electromagnetic interference (EMI) protection and/or shielding requirements. The ability to model and predict the electromagnetically (EM) induced currents coupled to UAV frames and wires, which propagate to their sense and/or control components, as it is in other modern electronic equipment, is a grand challenge to fully mitigate. To attack the full problem, there are too many input and state variables to treat computationally, especially when using brute force full-wave simulations [10], [11].

Numerous simulations and measurements need to be performed for a specific UAV to exhaustively quantify the coupled currents' variations due to the various flying conditions and possible environments. That is, the prediction of the coupled current to the UAV is complicated by the permutations of its orientation relative to the incident field, the polarization of the incident field, the operating frequency, etc. Even simple wire arrangements exhibit wide variations in the induced current with respect to the frequency, polarization, and direction of the incident wave [12]. These variations generate a
TABLE I

COMPARISON BETWEEN THIS WORK AND PREVIOUSLY REPORTED CMA COUPLING RESEARCH

\begin{tabular}{|c|c|c|c|c|}
\hline REF. & $\begin{array}{l}\text { SCATTERER } \\
\text { COMPLEXITY }\end{array}$ & $\begin{array}{l}\text { NO. OF } \\
\text { MODES }\end{array}$ & $\begin{array}{l}\text { ORIENTATION } \\
\text { SENSITIVITY }\end{array}$ & $\begin{array}{l}\text { EXPERIMENTAL } \\
\text { VERIFICATIONS }\end{array}$ \\
\hline [23] & $\begin{array}{c}\text { Simple } \\
\text { (rectangular box) }\end{array}$ & 4 & $\mathrm{NO}$ & YES \\
\hline [24] & $\begin{array}{c}\text { Simple } \\
\text { (rectangular box } \\
\text { with slot) }\end{array}$ & 10 & NO & NO \\
\hline [25] & $\begin{array}{c}\text { Medium } \\
\text { (PCB connected to } \\
\text { cable) }\end{array}$ & 3 & NO & YES \\
\hline \multirow[t]{3}{*}{ [26] } & $\begin{array}{c}\text { Simple } \\
\text { (flat circuit loop) }\end{array}$ & 4 & NO & NO \\
\hline & $\begin{array}{c}\text { Simple } \\
\text { (bent PCB) }\end{array}$ & 6 & NO & NO \\
\hline & $\begin{array}{c}\text { Simple } \\
\text { (Heatsink model as } \\
\text { metallic box) }\end{array}$ & 6 & NO & NO \\
\hline [27] & $\begin{array}{c}\text { Simple } \\
\text { (High speed } \\
\text { connector) }\end{array}$ & 10 & NO & NO \\
\hline [28] & Heatsink & 5 & NO & YES \\
\hline [29] & $\begin{array}{c}\text { Complex } \\
\text { (Car excited by } \\
\text { monopole) }\end{array}$ & 10 & YES & NO \\
\hline $\begin{array}{l}\text { This } \\
\text { work }\end{array}$ & $\begin{array}{c}\text { Complex } \\
\text { (UAV model) }\end{array}$ & 11 & YES & YES \\
\hline
\end{tabular}

need for computational and experimental tools to predict and quantify the variations in coupling with the incident wave's polarization, direction, and frequency.

To meet this need, we adapt the Characteristic Mode Analysis (CMA) to quantify the coupled current to a simplified UAV model. CMA is a theory that decomposes the currents induced on a scatterer in terms of a set of fundamental independent modes and provides the relative significance of each mode at the frequency range of interest [13]. CMA has been extensively employed in the design of antennas and in the analysis of the electromagnetic scattering characteristics of various nanostructures [14]-[17] Moreover, CMA has been previously employed for studying the use of the frame of a UAV as an antenna [18], [19]. We recently used CMA to study coupling and interference to wires and other simple structures [20]-[22]. But, to the best of our knowledge, this is the first experimentally verified analysis of coupling and interference to 
a UAV using CMA. However, Table I summarizes relevant previously reported CMA studies and compares them to this work. Most of the papers in Table I, studied the total radiated power by the scatterer, therefore, the scatterer has to be connected to an external source which is not the goal of this analysis, where coupling due to an incident plane wave is the main focus. Cao et al., on the other hand, studied the interaction between a monopole antenna and a car frame and how the interaction affects the radiation patterns of a car frame [29]. Cao et al. illustrated that the excitation source direction is crucial for estimating the excited modes of the scatterer. However, no analysis of the variation in the coupled current with the variations in the incident wave directions was reported. Moreover, no direct experimental measurements of the coupled current using a current probe were previously reported.

In summary, comparing the work presented in this paper with the studies in Table I shows the novelty of this work in terms of the novel scatterer considered, the relatively large number of studied modes, and the novel experimental verifications of CMA predictions.

In this paper, a quadcopter UAV and its subsystems are represented by a simplified model that consists of 4 wires attached to a square metallic patch, as shown in Fig. 1. The model was designed to represent the metallic parts typically found in a UAV since the metallic parts dominate the electromagnetic response [30]. Each of the four wires represents the fusion of all the cables commonly found in the arms of a quadcopter, whereas the square metallic patch represents the ground plane of the controller or electronic circuitry typically found at the center of a quadcopter. As will be emphasized in this paper, the fusion of the wires with the square metallic patch generates a modal behavior that is significantly different from that of wire or a patch by itself. The UAV model is simple enough to allow the quantitative assessment of the coupled current using both full-wave simulations and experimental measurements. Yet, the model is complex enough to generate conclusions that can be applied to a wide range of quadcopters with similar symmetries in shape.

CMA was applied to calculate the fundamental modes of the proposed UAV model and the characteristics of these modes. We use these modes to predict and explain the coupled current calculated at different locations on the UAV model due to plane wave excitations at different incident directions. The CMA predictions were validated using unique experimental measurements of the coupled current. Many experimental measurements require homogenous Transverse Electromagnetic Wave propagation from the source of illumination to the device under test (DUT) to isolate the effect of the angle of incidence or the DUT orientation on the coupled current. Field homogeneity cannot be easily achieved in a semianechoic chamber or a reverberation chamber due to multipath reflections. The Gigahertz Transverse Electromagnetic Cell (GTEM) is a common test environment that can administer a largely homogenous electromagnetic field across a broad bandwidth. GTEMs have been efficiently evoked in the EMI analysis of PCBs, cables, and other devices [31]-[34]. The CMA-based predictions for the proposed model were validated through experimental measurements in a GTEM. The CMA predictions, full-wave simulations, and the experimentally

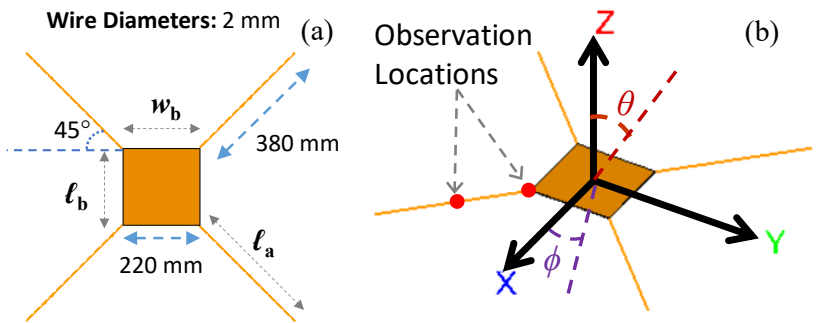

Fig. 1. (a) A sketch of the quadcopter UAV model showing the general dimensions of the structure, (b) A sketch of the model showing the UAV model orientation with respect to the angles $\theta$ and $\phi$.

measured values are then compared to demonstrate the approach's fidelity to build a low overhead predictive model.

This paper is organized as follows. Section II describes the simplified UAV model. Section III summarizes the CMA theory and how it is adapted for the analysis of coupling and interference. Section III presents in detail the CMA predictions of the currents coupled to the UAV model. Section IV describes the experimental setup inside a GTEM, and Section V discusses the measured coupled currents and the validation of the CMA predictions. Section VI summarizes the Conclusions and Future Work.

\section{SIMPLIFIED UAV MODEL}

The simplified UAV model is shown in Fig. 1a. The proposed model consists of a square patch with a $220 \mathrm{~mm}$ edge length and four wires attached at the patch's corners. The four wires of the model make an angle of $45^{\circ}$ with the edge of the square patch. The four identical wires represent the wire connections between the controller and the motors of a quadcopter UAV. The wires' lengths and radii are $380 \mathrm{~mm}$ and $0.5 \mathrm{~mm}$, respectively. In UAVs, the wires might have larger radii but we found that the radius of the wire has a negligible effect on the following results as long as the aspect ratio for the wire is high enough to maintain the thin wire configuration. A perfect electric conductor boundary condition is assigned to all components of the model in Fig. 1a.

The proposed UAV model was excited by a $150 \mathrm{mV} / \mathrm{m}$ incident plane wave at multiple angles of incidence, $\theta$, and $\phi$, as defined in Fig. $1 \mathrm{~b}$ while the polarization angle is zero for all the following results. The coupled current varies with the observation location on the UAV model. To highlight these variations, current monitors were placed at two locations on the UAV model: $(i)$ the edges of one of the four wires, where it meets the corner of the square patch, and (ii) the middle of the wire as shown in Fig. 1b. The two observation locations will be termed corner and middle, respectively, for the remainder of the manuscript.

The UAV model was simulated using the full-wave Method of Moments (MOM) solver FEKO [35] and the simulated coupled currents are shown in Fig. 2. Fig. 2 shows the coupled current versus frequency simulated at 2701 different angles of incidence, $\theta$, and $\phi$ where $\theta$ varies from $0^{\circ}$ to $180^{\circ}$ in a $5^{\circ}$ step and $\phi$ varies from $0^{\circ}$ to $360^{\circ}$ in a $5^{\circ}$ step to cover the entire sphere covering the UAV model while the polarization angle is kept zero for all the analysis. Fig. 2a shows the coupled current at the corner, and Fig. $2 \mathrm{~b}$ shows the current at the middle 

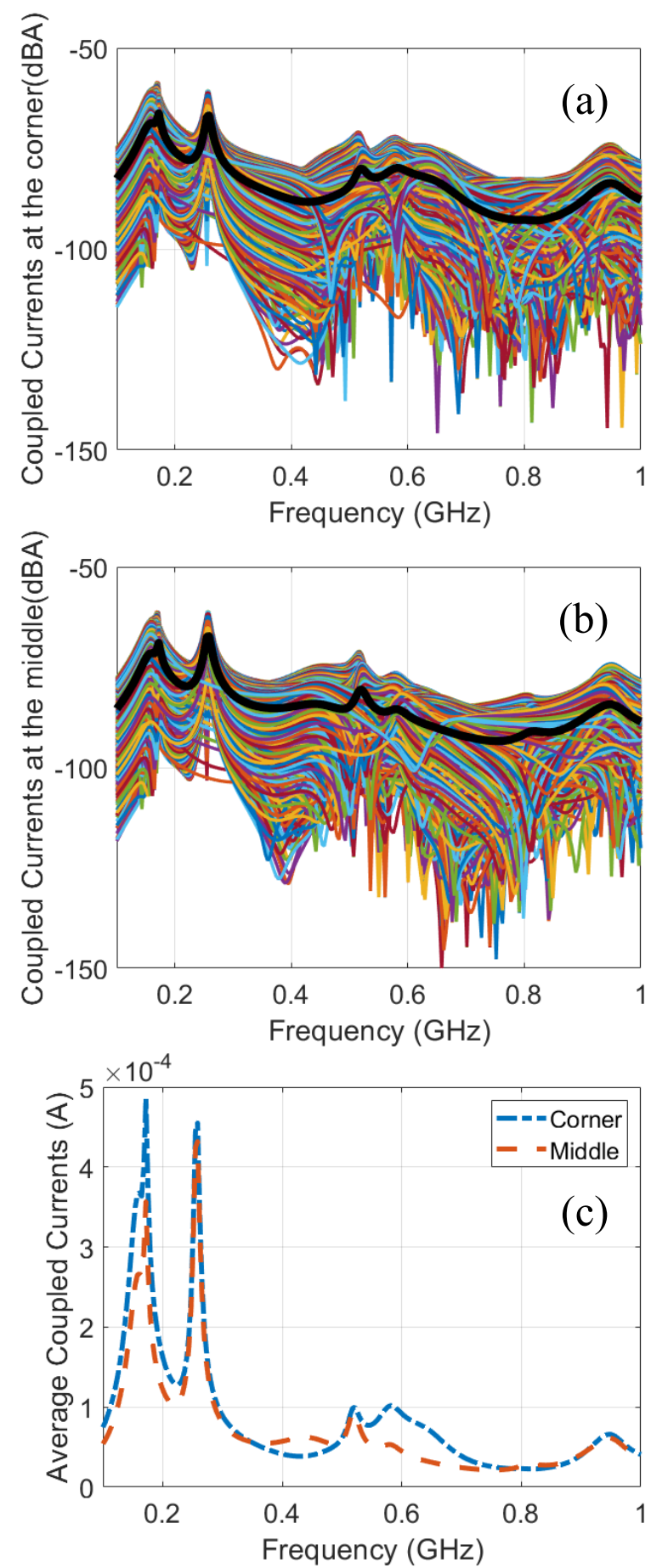

Fig. 2. The FEKO simulated coupled current to the UAV model at 2701 different angles of incidence $\theta$ and $\phi$ measured at (a) the edge of the wire, (b) the middle of the wire. (c) The coupled current averaged at all incidence orientations.

observation locations of the same wire. Each colored curve in Fig. 2 represents a different incident direction, i.e. a different $\{\theta, \phi\}$ value. The black curves in Fig. 2a and Fig. 2b represent the average coupled current at each location versus frequency. The coupled current exhibits significant variations with respect to the frequency and angles of incidence. The coupled current can vary by more than $70 \mathrm{~dB}$ based on the angle of incidence. The coupled current can even approach zero at some incidence angles, which is not shown in the log-scale curve in Fig. 2a and
Fig. 2b. Fig. 2c shows the coupled current, averaged with respect to incidence angles, at the two observation locations. That is, Fig. 2c shows the black curves in Fig. 2a and Fig. 2b in linear scale. Clearly, the coupled current differs with respect to the observation location.

Fig. 2 shows several minima and maxima in the coupled current at various frequencies, and additional maxima or minima might emerge if more angles of incidence are considered. It is also hard to predict the incidence angles that maximize the coupling current or the frequency range where the coupled current is maximum on average. In the following Section, the CMA theory is briefly summarized to show how it can facilitate predicting the coupled current at arbitrary frequencies and incidence angles.

\section{THEORY OF CHARACTERISTIC MODES ANALYSIS}

CMA is a full-wave electromagnetic technique that decomposes the total surface current coupled to a scatterer into a set of fundamental modes, called eigen-currents, and calculates the relative importance of each mode at any frequency [36]. The modes can be calculated using the following eigenvalue equation [13]:

$$
\mathbf{X}\left(\mathbf{J}_{\mathbf{n}}\right)=\lambda_{n} \mathbf{R}\left(\mathbf{J}_{\mathbf{n}}\right)
$$

where $\mathbf{X}$ and $\mathbf{R}$ are the imaginary and real parts of the MOM impedance matrix of the UAV model, respectively; $\mathbf{J}_{\mathbf{n}}$ is the eigen-current, and $\lambda_{n}$ is the eigenvalue of mode $n$. Equation (1) above is solved at every frequency to generate the corresponding $\mathbf{J}_{\mathbf{n}}$ and $\lambda_{n}$ values. At any frequency, the total current coupled to a scatterer due to an incident field can be expressed as [37]:

$$
\mathbf{I}=\sum_{n} \frac{V_{n} \mathbf{J}_{\mathbf{n}}}{\left(1+j \lambda_{n}\right)}
$$

where $V_{n}$ is the modal excitation coefficient that represents the coupling between the incident fields and mode $n$. The modal excitation coefficient $V_{\mathrm{n}}$ can be calculated using the following integral over the outer surface of the scatterer [37]:

$$
V_{n}=\iint \mathbf{J}_{\mathbf{n}} \cdot \mathbf{E}^{\mathbf{i}} d s
$$

where $\mathbf{E}^{\mathbf{i}}$ is the incident electric field.

To predict the coupling to any system using CMA, the following parameters should be studied in the following order:

1. Modal significance $\left(M S_{n}\right)$ : identifies the relative weight of mode $n$, i.e., it describes the significance of its contribution to the scatterer's total coupled current. It can be calculated as: $M S_{n}=\frac{1}{\left|1+j \lambda_{n}\right|}$. The $M S_{n}$ has a maximum value of 1 , which occurs when the eigenvalue $\lambda_{n}$ has a value of zero. At any frequency of interest, the ratio between the different modes' MSn values allows us to identify the modes that dominate the coupled current. The $M S_{\mathrm{n}}$ is independent of the excitation, and it only depends 
on the shape, size, and material properties of the scatterer and its environment.

2. Eigen-currents or modal currents $\mathbf{J}_{\mathbf{n}}$ : identify the current distribution of each mode $n$. The modal currents will illustrate the maximum and minimum current locations of each mode. The total current, I, coupled to the scatterer, is a weighted sum of these eigen-currents $\mathbf{J}_{\mathbf{n}}$. Therefore, knowing $\mathbf{J}_{\mathbf{n}}$ 's maximum/minimum locations will facilitate predicting the locations where $\mathbf{I}$ is maximum or minimum. The eigen-currents $\mathbf{J}_{\mathbf{n}}$ are also completely independent of the excitation.

3. Modal excitation coefficient $V_{n}$ : represents the coupling between the incident field and mode $n$ of the scatterer. The modal excitation coefficient $V_{n}$ shows which angles of incidence maximize coupling to mode $n$ and which angles of incidence will cause mode $n$ not to be expressed at all in the total current $\mathbf{I}$.

It is important to re-emphasize that the CMA parameters $M S_{n}$ and $\mathbf{J}_{\mathbf{n}}$ are independent of the incident field. The dependency on the excitation is entirely encapsulated in the modal excitation coefficient $V_{\mathrm{n}}$. As a result, CMA will give an insight into all the supported modes of the structure and how to excite each mode without the need of testing all possible angles of incidence to make sure that all the modes that the scatterer can support are expressed. Therefore, once the $M S_{n}$ and $\mathbf{J}_{\mathbf{n}}$ parameters are calculated, the computational time needed to quantify coupling to the scatterer with respect to the frequency and angles of incidence of the excitation can be highly optimized, as will be highlighted in the following Section.

\section{CMA OF UAV MODEL}

Using the full-wave solver FEKO, we performed the CMA of the UAV model in Fig. 1 over the frequency range that varies between $0.1 \mathrm{GHz}$ and $1 \mathrm{GHz}$. The modal significances, $M S_{n}$, of the UAV model are shown in Fig. 3. The modal significance spectrum in Fig. 3 shows all the possible coupling pathways to the UAV model. The peaks or the resonance frequencies of the modes represent the frequencies where the coupled current can reach maximum values. For example, Modes 1 and 2 resonate at $0.17 \mathrm{GHz}$, Mode 3 resonates at $0.19 \mathrm{GHz}$, and Mode 4 resonates at $0.24 \mathrm{GHz}$. These are the critical frequencies for the UAV mode in Fig. 1 for the frequency range up to $0.4 \mathrm{GHz}$. Therefore, the first advantage of the CMA is that it identifies for us the critical frequencies of the Device Under Test (DUT). Therefore, instead of testing the structure at hundreds of frequencies, we can only focus on the frequencies where the modes resonate.

It is important to emphasize that the coupled current, for $a$ specific excitation at a particular angle of incidence, will not show all the peaks in the $M S_{\mathrm{n}}$ spectrum in Fig. 3. Different modes are excited at different angles of incidence, as will be described in the following paragraph. However, the $M S_{n}$ spectrum identifies quantitatively, with no trial and error, the critical frequencies, where we need to focus our full-wave simulations and experimental measurements. Moreover, the frequency range where the modes' density is rare or where the $M S_{\mathrm{n}}$ does not reach high values is the frequency range where

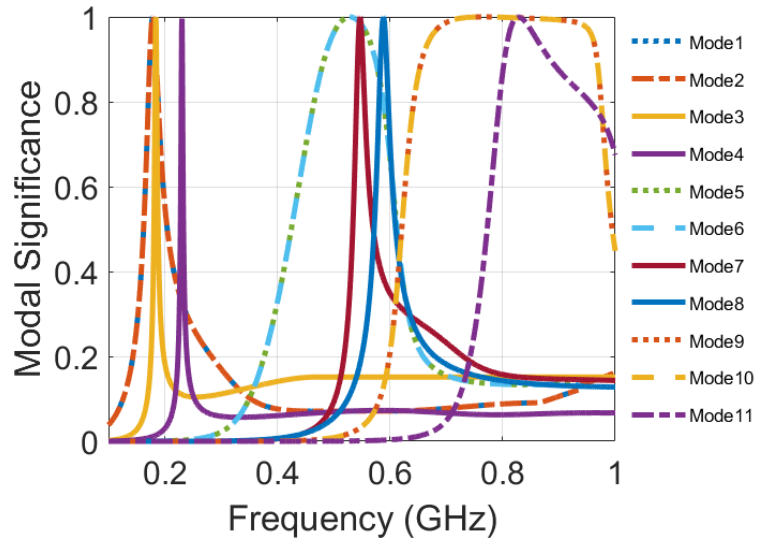

Fig. 3. Modal significance of the first 11 modes of the proposed UAV model in Fig. 1.

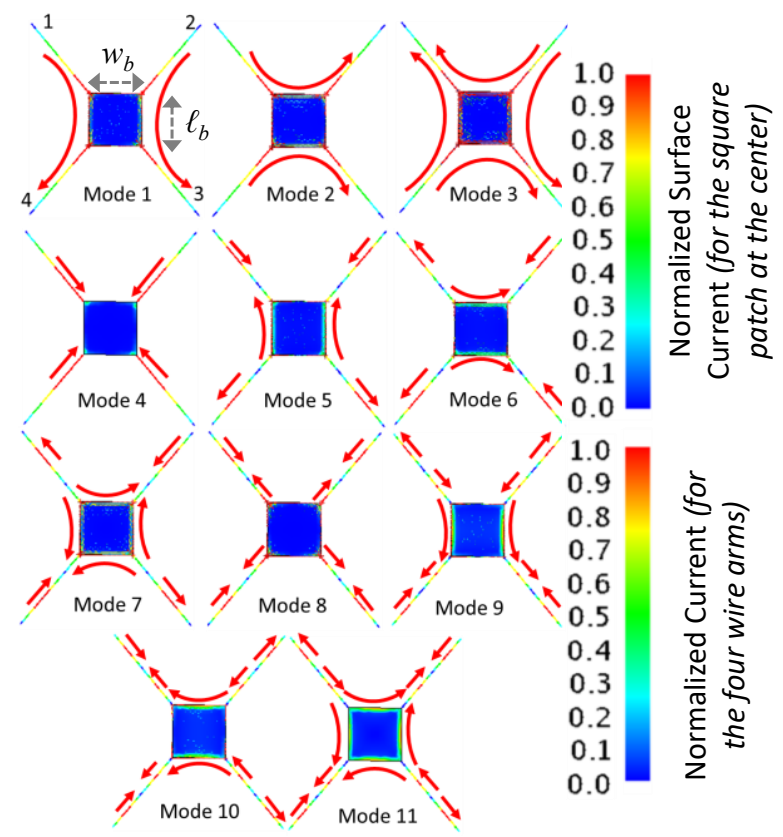

Fig. 4. Normalized eigen-current distribution of the first 11 modes of the UAV structure. Each eigen-current distribution is plotted at the resonance frequency of its mode.

the coupled current will be lower on average than other frequency ranges of the same bandwidth. For example, the frequency range between $0.26 \mathrm{GHz}$ and $0.39 \mathrm{GHz}$ does not have any resonating modes. Therefore, the average coupled current in this frequency range should be lower than that at slightly lower or slightly higher frequencies, which is confirmed in Fig. 2 at all observation locations. Therefore, the observation of $M S_{n}$ is more useful for identifying where the coupling is unlikely to occur rather than where the coupling is likely to occur, in the sense that having a resonant mode doesn't mean that the incident field can excite this mode, or that the mode will exhibit meaningful current at the location of interest as will be discussed in the following paragraphs. All the previous conclusions are achieved directly from Fig. 3 without testing hundreds of different angles of incidence.

The eigen-current distributions $\mathbf{J}_{\mathbf{n}}$ of the UAV model are shown in Fig. 4. Each eigen-current distribution in Fig. 4 is 
plotted at the resonance frequency of its mode. The eigencurrent distribution in Fig. 4 is normalized with respect to the maximum current value of each mode. The red arrows in Fig. 4 represent the general trend of the current of each mode or eigencurrent, whereas the colors show the exact distribution. The hotspots of the modal currents, i.e., the locations where the modal currents are maximum, are shown in red, whereas the locations where the modal currents are zero are shown in blue. For example, Mode 1 has a hotspot at the corner observation location, whereas Mode 9 has a minimum at both the corner and middle observation locations.

The UAV model in Fig. 1, and many quadcopters, has 4-fold symmetry. This symmetry is reflected in the current distribution of the eigen-currents or modes shown in Fig. 4. For example, Mode 2 is identical to Mode 1 if it is rotated by $90^{\circ}$. Similarly, Mode 5 is identical to Mode 6, and Mode 9 is identical to Mode 10, except for a $90^{\circ}$ rotation, as shown in Fig. 4. Due to this symmetry, the three previously mentioned mode pairs have identical $M S_{\mathrm{n}}$, as shown in Fig. 3. That is, $M S_{1}=M S_{2}, M S_{5}=$ $M S_{6}$, and $M S_{9}=M S_{10}$. This means that Mode 1 and Mode 2 will resonate at the same frequency. If the symmetry is broken, then the previous mode pairs will have different modal significance. On the other hand, the current distribution of Mode 3 is a merge of Mode 1 and Mode 2, as shown in Fig. 4, and it resonates at a slightly higher frequency as shown in Fig. 3. This behavior was previously reported in similar but simpler structures that had 4fold symmetry [38], [39]. However, combining the 4 wires to the square plate leads to the emergence of new modes that were not easy to predict from the individual modes of the wire or the individual modes of the square plate.

The Modal Excitation Coefficients, $\mathrm{V}_{\mathrm{n}}$ can be calculated by using transverse electromagnetic plane wave at different angles of incidence, identifying the incident electric field due to this excitation, $\mathbf{E}^{\mathbf{i}}$, and recalculating the modal excitation coefficient $\mathrm{V}_{\mathrm{n}}$ according to (3). The Modal Excitation Coefficients, $\mathrm{V}_{\mathrm{n}}$ of the first 11 modes, which by reciprocity can be estimated using the radiation characteristics of each mode, are shown in the polar plots in Fig. 5. The radiation pattern of each mode in Fig. 5 is plotted at the resonance frequency of this mode and normalized to its maximum value. All the radiation patterns in Fig. 5 are in the $\theta$ plane with a fixed $\phi$ value of $0^{\circ}$ and a fixed polarization angle of $0^{\circ}$. Angles where the modal excitation coefficient is maximum represent the optimum direction to excite a particular mode. Angles of zero modal excitation coefficient in Fig. 5 indicate incident directions that cannot excite the mode regardless of its significance at a particular frequency. For example, Mode 4 cannot be excited at any frequency at $\theta=0^{\circ}$ or at $\theta=90^{\circ}$ for $\phi=0^{\circ}$, and it can be optimally excited when the incidence angle is at $\theta=45^{\circ}$.

Most of the 11 modes are narrowband modes. Hence, the changes in the eigen-current distribution of a mode within the frequency band where a mode is significant can be neglected. For example, Fig. 6 presents the eigen-current distribution and the radiation characteristics of Mode 5 at three different frequencies within the bandwidth of Mode 5, i.e at three frequencies where $\mathrm{MS}_{5}$ is larger than 0.707. The current distribution of Mode 5 and its radiation pattern doesn't change dramatically within the bandwidth of the mode. That is, the
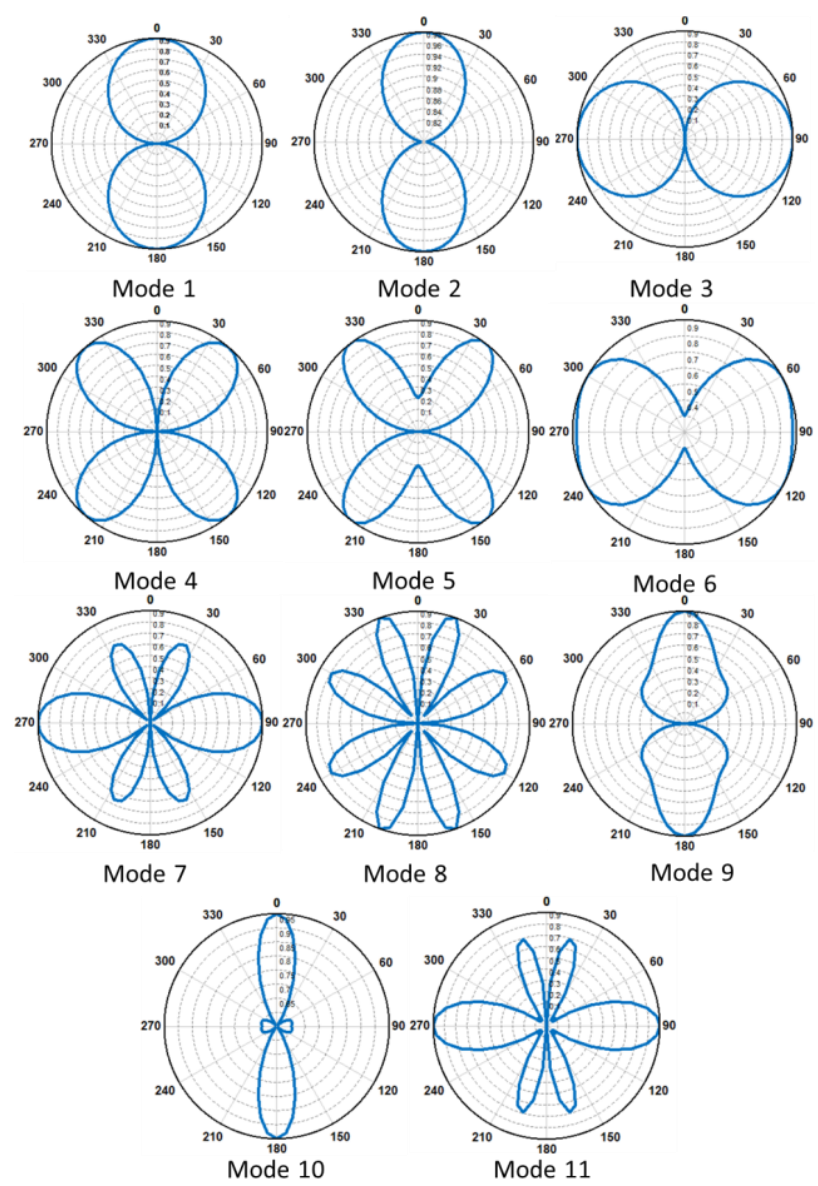

Fig. 5. Normalized Modal Fields of the first 11 modes of the UAV model. The radiation pattern of each mode is plotted at the resonance frequency of this mode.

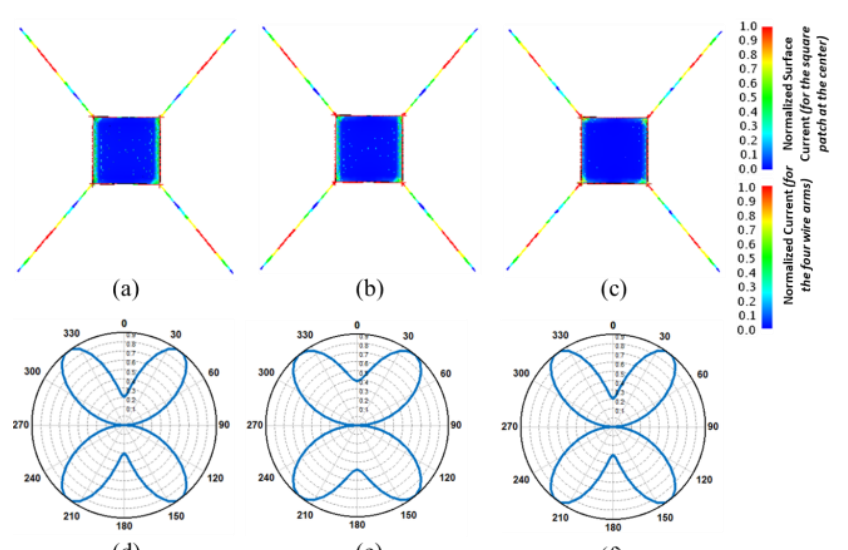

(d)

(e)

(f)

Fig. 6: Eigen-current distribution of Mode 5 of the UAV structure at: (a) $490 \mathrm{MHz}$, (b) $520 \mathrm{MHz}$, (c) $580 \mathrm{MHz}$. Normalized Modal Fields of Mode 3 of the UAV model at: (d) $490 \mathrm{MHz}$, (e) $520 \mathrm{MHz}$, (f) $580 \mathrm{MHz}$.

magnitude of the modal current slightly changes with frequency but the hotspot locations are maintained, and therefore, the radiation pattern of the modes doesn't change significantly as shown in Fig. 6. For frequencies that are significantly larger or smaller than the resonance frequencies of the mode, the eigencurrents and the radiation characteristics of a mode might show significant differences from the patterns at resonance. However, 
at these frequencies, the $\mathrm{MS}_{\mathrm{n}}$ of the modes will be too low to provide any significant contribution to the total coupled current.

The CMA information presented in Fig. 3 to Fig. 5 allows us to understand how a wide range of excitations might couple to the UAV. For example, at any frequency of interest, Fig. 3 can identify the significant modes in this frequency range. By investigating Fig. 4, we can determine whether these significant modes have nonzero currents at the location of interest. Next, by examining Fig. 5, we can identify the incident directions that will excite these modes. These will be the incident directions where the coupled current can be maximized. Then by performing the summation in (2), we can predict the coupled current.

On the other hand, if the angle of incidence of the excitation is known, we can predict the modes that will be excited. Based on their modal significance in Fig. 3, we can predict the frequencies where coupled current resonates. For example, consider the case where we are interested in the current coupled to the corner observation location when the plane wave excitation is incident at $\theta=0^{\circ}$ and $\phi=0^{\circ}$. This direction of incidence is only capable of exciting Modes 1, 2, 9, and 10. By examining these Modes' current distributions, shown in Fig. 4, we can see that only Modes 1 and Mode 2 have hotspots, shown in red, at the corner observation location. Modes 9 and 10 have minimal current, shown in blue, at this location. Therefore, we expect the total coupled current to show peaks only at the resonance frequencies of Modes 1 and 2, shown to be around $0.17 \mathrm{GHz}$ in Fig. 3. Fig. 7 confirms these predictions by showing the full-wave simulated current at the corner observation location due to a plane wave excitation at $\theta=0^{\circ}$. A clear peak is present in the coupled current in Fig. 7 near the frequency of $0.17 \mathrm{GHz}$, which is the same resonance frequency of Modes 1 and 2. Fig. 7 also shows a strong minimum near $0.77 \mathrm{GHz}$. This minimum is not due to the absence of current coupling pathways because Modes 9,10 , and 11 are significant at this frequency. The minimum in the coupled current at 0.77 $\mathrm{GHz}$ exists because these 3 modes contribute zero current at the corner observation location. All of these predictions were achieved from the CMA information generated in Fig. 3 to Fig. 5. The following Section describes how varying the UAV model dimensions can affect the modal behavior previously described.

\section{Sensitivity of Modes to UAV Model Dimensions}

The dimensions of a UAV can vary in different models. Therefore, in this Section, we explore how the modal characteristics vary with the dimensions of the UAV model. Three dimensions of the UAV model in Fig. 1a are varied: $\ell_{\mathrm{a}}$, $\ell_{\mathrm{b}}$, and $w_{\mathrm{b}}$. The effect of varying the UAV's arm length $\ell_{\mathrm{a}}$ on the modal behavior, for fixed center body dimensions $\ell_{\mathrm{b}}=w_{\mathrm{b}}=220$ $\mathrm{mm}$, is plotted in Fig. 8a. For this analysis, the arm length $\ell_{\mathrm{a}}$ is increased from $200 \mathrm{~mm}$ to $500 \mathrm{~mm}$ in $50 \mathrm{~mm}$ increments. For each $\ell_{\mathrm{a}}$ value, we recalculated the modal significance, $M S_{\mathrm{n}}$, of the modes, and we plotted the resonance frequency of $M S_{1}-$ $\mathrm{MS}_{3}$ in Fig, 8a. The analysis in Fig. 8a shows that the resonance frequencies of Modes 1-3 decrease proportional to $\sim 1 / \ell_{\mathrm{a}}$.

The effect of varying the patch's length, $\ell_{\mathrm{b}}$, for a fixed arm length and patch width, $\ell_{\mathrm{a}}=380 \mathrm{~mm}$ and $w_{\mathrm{b}}=220 \mathrm{~mm}$, is shown

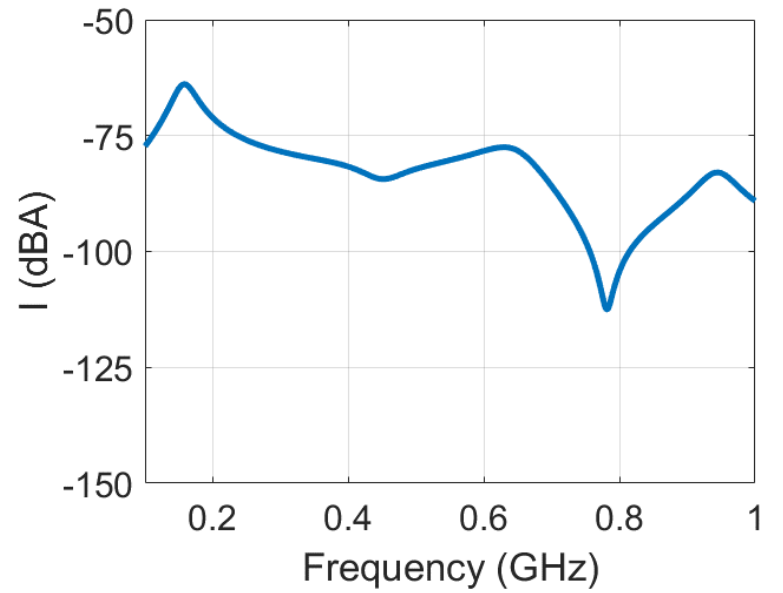

Fig. 7. Coupled current to the edge of the wire due to an incident wave at $\theta=0^{\circ}$.
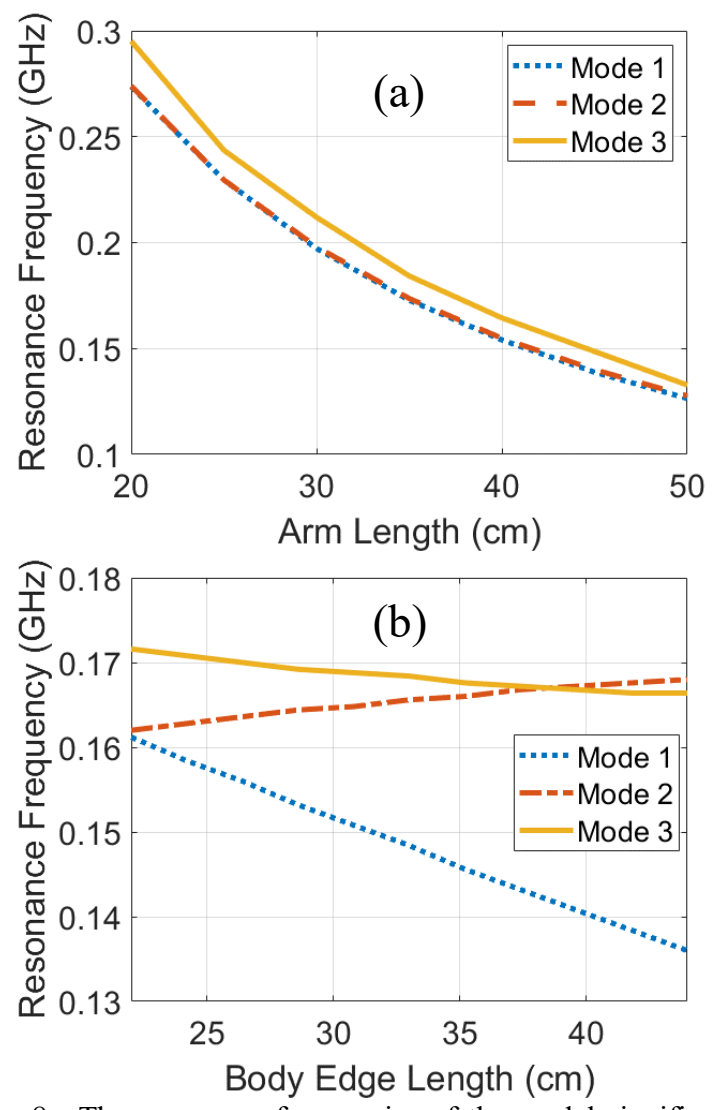

Fig. 8. The resonance frequencies of the modal significance of Modes 1-3 when (a) the arm length $\ell$ a was varied and (b) when the body length $\ell b$ was varied.

in Fig. 8b. As the body length $\ell_{b}$ increases from $220 \mathrm{~mm}$ to 440 $\mathrm{mm}$ in $22 \mathrm{~mm}$ increments, the center patch becomes a rectangle instead of a square breaking the 4-fold symmetry. Therefore, instead of Mode 1 and Mode 2 showing identical resonance frequencies, Fig. $8 \mathrm{~b}$ shows that as $\ell_{\mathrm{b}}$ is increased, Mode 1 and Mode 2 start showing opposite trends. That is, the resonance frequency of Mode 1 starts to decrease and the resonance frequency of Mode 2 starts to increase. The resonance frequency of Mode 1 is the most sensitive to the change in $\ell_{\mathbf{b}}$, decreasing from $0.162 \mathrm{GHz}$ to $0.136 \mathrm{GHz}$, which corresponds 
to a $15.5 \%$ reduction in the resonance frequency for a $100 \%$ increase in the $\ell_{\text {b. }}$. On the other hand, Mode 2 only shows a 3.5 $\%$ increase in its resonance frequency for a $100 \%$ increase $\ell_{\mathrm{b}}$. The current distribution of the modes shown in Fig. 4 explains this behavior because the current distribution of Mode 1 is parallel to $\ell_{\mathrm{b}}$, but the current distribution of Mode 2 is parallel to $w_{\mathrm{b}}$. Therefore, increasing $\ell_{\mathrm{b}}$ and keeping $w_{\mathrm{b}}$ constant has a marginal effect on Mode 2 but significantly affects Mode 1. Mode 3 is a hybrid mode combining the current distributions of Mode 1 and Mode 2, as shown in Fig. 4. Therefore, its resonance frequency follows approximately the average of the trends of Mode 1 and Mode 2. Since Mode 1 shows a more significant decrease than Mode 2, the overall trend of Mode 3 is a decrease with the increase in $\ell_{\mathrm{b}}$.

Therefore, another advantage of the CMA is that it explicitly delineates the current distribution of the modes. This current distribution will allow us to predict which modes will change their resonance frequencies and which modes will have their resonance frequencies unaffected with a change in the DUT's geometry. The mode resonances will be conveyed to the coupled current, which is a weighted summation of the modes as in (2). Now that the modal characteristics and behavior are fully quantified, the next Section shows how CMA can be used to predict the coupled current in experimental measurements.

\section{EXPerimental Testing OF the Proposed Model}

An EMCO 5317 GTEM was used for testing the CMA predictions. The GTEM cell has a maximum septum height of $1.5 \mathrm{~m}$ and a recommended frequency range of DC $-18 \mathrm{GHz}$. At its feed, the GTEM has a $50 \Omega 7 / 16$ DIN coaxial input. Port 1 of a Rohde \& Schwarz ZVA 24 vector network analyzer (VNA) was connected to this feed to generate the incident TEM wave. The GTEM is terminated with pyramidal foam absorbers and distributed resistive loads to prevent any reflections. The UAV model in Fig. 1 was built from copper wires and a square copper sheet. It was placed inside the GTEM at a $1.5 \mathrm{~m}$ septum height to be excited by the generated TEM fields, as shown in Fig. 9 . The field strength at the UAV location can be estimated by dividing the input voltage at the TEM-cell port by the septum height. A $225 \mathrm{mV}$ was applied at port 1 of the GTEM to stimulate it. Therefore, the field strength at the UAV location is $\sim 150 \mathrm{mV} / \mathrm{m}$. BCP-512 clamp-on broadband current probe with an operable frequency range of $1 \mathrm{MHz}-1 \mathrm{GHz}$ was placed at different locations on the UAV model to sample the total coupled current. The experimental setup in Fig. 9 allows us to validate the CMA predictions since the total coupled current is a weighted summation of the modes in Fig. 3-5. The current probe was connected to Port 2 of the VNA. The measured Sparameter, $S_{21}$, was converted to the coupled current through the following formula:

$\mathbf{I}(\mathrm{dBA})=$ Input voltage at the TEM-cell port $(\mathrm{dBV})+\mathrm{S} 21(\mathrm{~dB})$

- Probe Transfer Impedance (dB $\Omega$ ) - Cable Loss (dB)

In the first experimental measurement, the UAV model was oriented at normal incidence to the incident wave inside the GTEM, i.e., $\theta=0^{\circ}$ and $\phi=0^{\circ}$ and the polarization angle is zero. The current probe was placed at the corner location, and the coupled current was measured and compared to the simulations

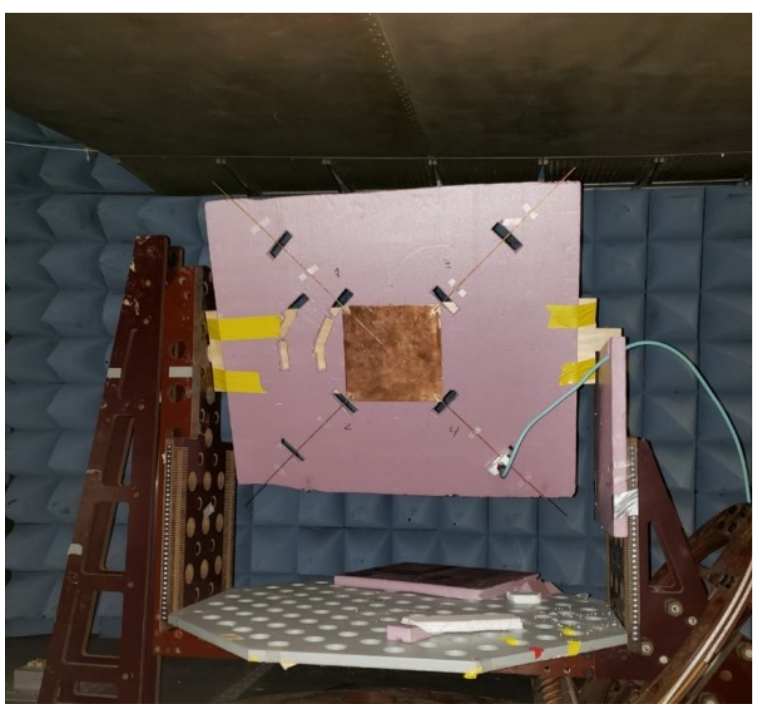

Fig. 9. Experimental setup showing the realized UAV model inside the GTEM and the current probe at one location.

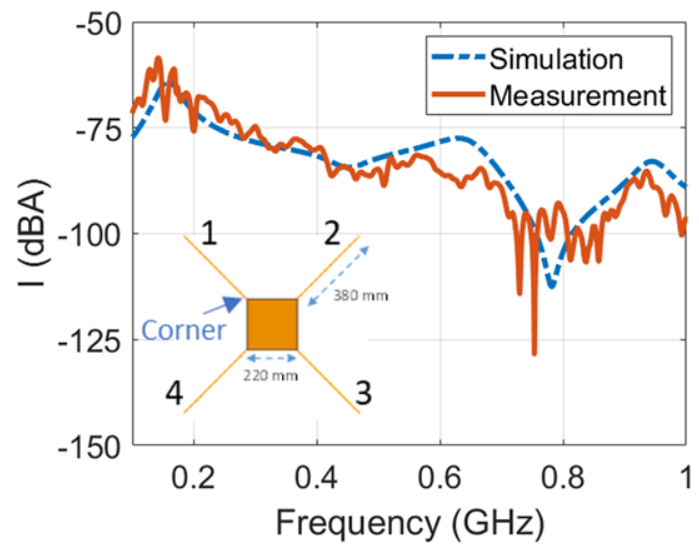

Fig. 10. Comparison between the measured and simulated currents at the edge of Wire 1 for normal incidence $\theta=0^{\circ}$ and $\phi=$ $0^{\circ}$.

that used incident plane waves with an electric field amplitude of $150 \mathrm{mV} / \mathrm{m}$. Excellent agreement was achieved between the measurements and the simulations, as shown in Fig. 10. For both the simulated and the measured current, the peak current was achieved near $0.17 \mathrm{GHz}$, and a minimum in the coupled current was experimentally observed near $0.77 \mathrm{GHz}$ providing validation of the CMA predictions.

To further test the CMA predictions, we re-examined the minimum in the coupled current near $0.77 \mathrm{GHz}$. Fig. 3 shows that there are significant modes near this frequency, e.g., Modes 9 and 10. However, Fig. 4 shows that these modes' current distributions have a minimum at the corner location. The maximum of their current distribution occurs at the middle location. Therefore, CMA predicts that this dip in the coupled current will disappear if the current probe is moved to the middle location. Fig. 11 shows the simulated and the measured current at the middle of the wire for the same UAV orientation. The dip in the coupled current near $0.77 \mathrm{GHz}$ is significantly reduced, validating the CMA prediction. Specifically, in Fig. 10 the minimum coupled current was less than $-115 \mathrm{dBA}$, but in Fig. 11, it was on the order of $\sim-90$ dBA. 


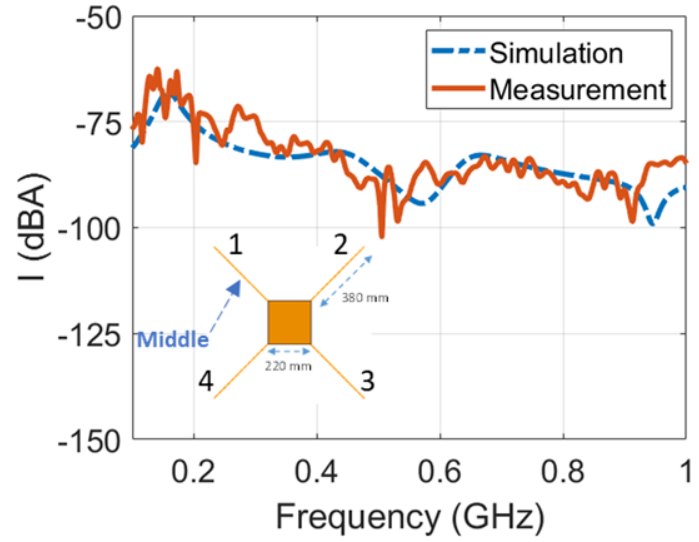

Fig. 11. Comparison between the measured and simulated currents at the middle of Wire 1 for normal incidence $\theta=0^{\circ}$ and $\phi$ $=0^{\circ}$.

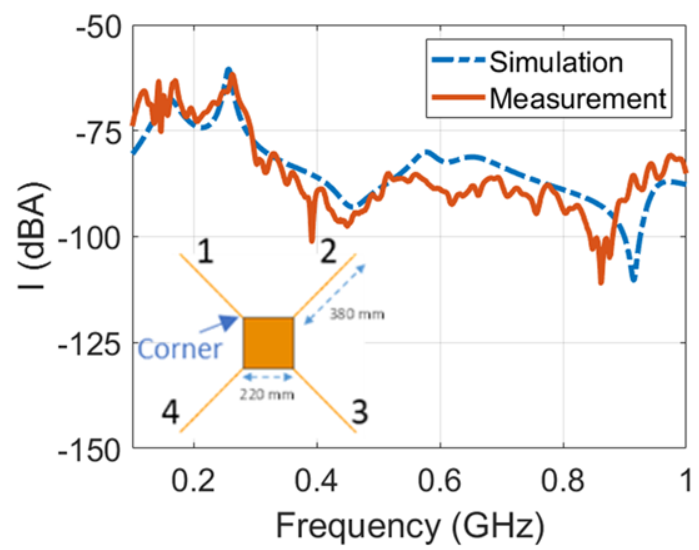

Fig. 12. Comparison between the measured and simulated currents at the edge of Wire 1 for oblique incidence $\theta=45^{\circ}$ and $\phi$ $=0^{\circ}$.

To further examine CMA's capabilities to predict coupled current, we examined Mode 4 which cannot be excited by normal incidence. Fig. 5 shows that the optimum angle to excite Mode 4 is $\theta=45^{\circ}$. Therefore, if we change the orientation of the incident wave relative to the UAV model, Mode 4 can be excited. We expect a new peak to emerge in the coupled current at its resonance frequency of $0.24 \mathrm{GHz}$, as shown in Fig. 3. Fig. 12 shows the coupled current at the corner location at an oblique incidence angle of $\theta=45^{\circ}$ and $\phi=0^{\circ}$ and polarization angle of zero. As predicted by CMA, a new peak emerges at $0.24 \mathrm{GHz}$ caused primarily by Mode 4 . Therefore, CMA provides a straightforward technique, without trial and error, to predict the peaks in the coupled current even if these peaks do not appear at all device orientations or angles of incidence.

In summary, we showed that the CMA could efficiently predict the coupled currents, at various incident angles, frequencies, and probe locations on a simplified but practical UAV model. These predictions were verified using full-wave simulations and experimental measurements. Therefore, CMA can significantly reduce the complexity of both computational simulations and experimental measurements of coupled and interfering currents in DUTs.

\section{CONCLUSIONS}

In this work, we developed a simple model for the wires and electronic circuitry of quadcopter Unmanned Aerial Vehicles (UAVs) using a square metallic patch and 4 wires. Characteristic Mode Analysis (CMA) was successfully applied to calculate the fundamental modes supported by the UAV model and the characteristics of these modes. The knowledge of this modal behavior facilitates the quantification of the UAV's electromagnetic susceptibility to external interference and allows us to identify the resonance frequencies that maximize the coupled current. Moreover, we showed that the CMA could predict the orientations that maximize the coupled current to the UAV model at a particular frequency. The CMA predictions were tested experimentally by building the UAV model, exciting it by plane waves inside a GTEM, and measuring the coupled current using a clamp-on current probe. Excellent agreement was achieved between the simulations and the measurements validating this approach for quantifying electromagnetic coupling and interference to UAVs and similar devices of interest. In the future, we will extend the CMA approach employed herein to study more complicated and practical UAV models.

\section{REFERENCES}

[1] B. I. Intelligence, "Drones are about to fill the skies within the next 5 years," Business Insider.

[2] P. Zhan, D. W. Casbeer, and A. L. Swindlehurst, "A Centralized Control Algorithm for Target Tracking with UAVs," in Conference Record of the Thirty-Ninth Asilomar Conference onSignals, Systems and Computers, 2005., Oct. 2005, pp. 1148-1152, doi: 10.1109/ACSSC.2005.1599940.

[3] J. R. Insua, S. A. Utsumi, and B. Basso, "Estimation of spatial and temporal variability of pasture growth and digestibility in grazing rotations coupling unmanned aerial vehicle (UAV) with crop simulation models," PLOS ONE, vol. 14, no. 3, p. e0212773, Mar. 2019, doi: 10.1371/journal.pone.0212773.

[4] J. Kim and J. P. Hespanha, "Cooperative radar jamming for groups of unmanned air vehicles," in 2004 43rd IEEE Conference on Decision and Control (CDC) (IEEE Cat. No.04CH37601), Dec. 2004, vol. 1, pp. 632-637 Vol.1, doi: 10.1109/CDC.2004.1428715.

[5] D. W. Casbeer, R. W. Beard, T. W. McLain, Sai-Ming Li, and R. K. Mehra, "Forest fire monitoring with multiple small UAVs," in Proceedings of the 2005, American Control Conference, 2005., Jun. 2005, pp. 3530-3535 vol. 5, doi: 10.1109/ACC.2005.1470520.

[6] Z. Xiao, P. Xia, and X. Xia, "Enabling UAV cellular with millimeterwave communication: potentials and approaches," IEEE Commun. Mag., vol. 54, no. 5, pp. 66-73, May 2016, doi: 10.1109/MCOM.2016.7470937.

[7] M. Mozaffari, W. Saad, M. Bennis, and M. Debbah, "Unmanned Aerial Vehicle With Underlaid Device-to-Device Communications: Performance and Tradeoffs," IEEE Trans. Wirel. Commun., vol. 15, no. 6, pp. 3949-3963, Jun. 2016, doi: 10.1109/TWC.2016.2531652.

[8] C. Zhan, Y. Zeng, and R. Zhang, "Energy-Efficient Data Collection in UAV Enabled Wireless Sensor Network," IEEE Wirel. Commun. Lett., vol. 7, no. 3, pp. 328-331, Jun. 2018, doi: 10.1109/LWC.2017.2776922.

[9] J. Lyu, Y. Zeng, R. Zhang, and T. J. Lim, "Placement Optimization of UAV-Mounted Mobile Base Stations," IEEE Commun. Lett., vol. 21, no. 3, pp. 604-607, Mar. 2017, doi: 10.1109/LCOMM.2016.2633248.

[10] T. Li, B. Wen, Y. Tian, Z. Li, and S. Wang, "Numerical Simulation and Experimental Analysis of Small Drone Rotor Blade Polarimetry Based on RCS and Micro-Doppler Signature," IEEE Antennas Wirel. Propag. Lett., vol. 18, no. 1, pp. 187-191, Jan. 2019, doi: 10.1109/LAWP.2018.2885373.

[11] A. V. Khristenko et al., "Magnitude and Spectrum of Electromagnetic Wave Scattered by Small Quadcopter in \$X\$ -Band," IEEE Trans. Antennas Propag., vol. 66, no. 4, pp. 1977-1984, Apr. 2018, doi: 10.1109/TAP.2018.2800640. 
[12] S. Kim, Y. Noh, J. Lee, J. Lee, J. Choi, and J. Yook, "Electromagnetic Signature of a Quadcopter Drone and Its Relationship With Coupling Mechanisms," IEEE Access, vol. 7, pp. 174764-174773, 2019, doi: 10.1109/ACCESS.2019.2956499.

[13] R. Harrington and J. Mautz, "Theory of characteristic modes for conducting bodies," IEEE Trans. Antennas Propag., vol. 19, no. 5, pp. 622-628, Sep. 1971, doi: 10.1109/TAP.1971.1139999.

[14] M. Cabedo-Fabres, E. Antonino-Daviu, A. Valero-Nogueira, and M. F. Bataller, "The Theory of Characteristic Modes Revisited: A Contribution to the Design of Antennas for Modern Applications," IEEE Antennas Propag. Mag., vol. 49, no. 5, pp. 52-68, Oct. 2007, doi: 10.1109/MAP.2007.4395295.

[15] S. Dey, D. Chatterjee, E. J. Garboczi, and A. M. Hassan, "Plasmonic Nanoantenna Optimization Using Characteristic Mode Analysis," IEEE Trans. Antennas Propag., vol. 68, no. 1, pp. 43-53, Jan. 2020, doi: 10.1109/TAP.2019.2938705.

[16] K. C. Durbhakula et al., "Electromagnetic Scattering From Individual Crumpled Graphene Flakes: A Characteristic Modes Approach," IEEE Trans. Antennas Propag., vol. 65, no. 11, pp. 6035-6047, Nov. 2017, doi: 10.1109/TAP.2017.2752218.

[17] A. M. Hassan, F. Vargas-Lara, J. F. Douglas, and E. J. Garboczi, "Electromagnetic Resonances of Individual Single-Walled Carbon Nanotubes With Realistic Shapes: A Characteristic Modes Approach," IEEE Trans. Antennas Propag., vol. 64, no. 7, pp. 2743-2757, Jul. 2016, doi: 10.1109/TAP.2016.2526046.

[18] Y. Chen and C. Wang, "Electrically Small UAV Antenna Design Using Characteristic Modes," IEEE Trans. Antennas Propag., vol. 62, no. 2, pp. 535-545, Feb. 2014, doi: 10.1109/TAP.2013.2289999.

[19] S. Sow, L. Guo, S. Zhou, and T. Chio, "Electrically small structural antenna design for small UAV based on characteristics modes," in 2017 11th European Conference on Antennas and Propagation (EUCAP), Mar. 2017, pp. 2134-2138, doi: 10.23919/EuCAP.2017.7928206.

[20] M. Z. M. Hamdalla, A. N. Caruso, and A. M. Hassan, "Predicting Electromagnetic Interference to a Terminated Wire Using Characteristic Mode Analysis," in 2020 International Applied Computational Electromagnetics Society Symposium (ACES), Jul. 2020, pp. 1-2, doi: 10.23919/ACES49320.2020.9196133.

[21] M. Hamdalla, B. Bissen, A. N. Caruso, and M. Hassan, "Experimental Validations of Characteristic Mode Analysis Predictions Using GTEM," presented at the IEEE International Symposium on Antennas and Propagation and USNC-URSI Radio Science Meeting, Montréal, Québec, Canada, Jul. 2020.

[22] M. Z. M. Hamdalla, A. M. Hassan, and A. N. Caruso, "Characteristic Mode Analysis of the Effect of the UAV Frame Material on Coupling and Interference," in 2019 IEEE International Symposium on Antennas and Propagation and USNC-URSI Radio Science Meeting, Jul. 2019, pp. 1497-1498, doi: 10.1109/APUSNCURSINRSM.2019.8888344.

[23] F. A. Dicandia, S. Genovesi, and A. Monorchio, "Efficient Excitation of Characteristic Modes for Radiation Pattern Control by Using a Novel Balanced Inductive Coupling Element," IEEE Trans. Antennas Propag., vol. 66, no. 3, pp. 1102-1113, Mar. 2018, doi: 10.1109/TAP.2018.2790046.

[24] S. Ghosal, A. De, A. P. Duffy, and A. Chakrabarty, "Selection of Dominant Characteristic Modes," IEEE Trans. Electromagn. Compat., vol. 62, no. 2, pp. 451-460, Apr. 2020, doi: 10.1109/TEMC.2019.2909932.

[25] Y. S. Cao, Y. Wang, L. Jiang, A. E. Ruehli, J. Fan, and J. L. Drewniak, "Quantifying EMI: A Methodology for Determining and Quantifying Radiation for Practical Design Guidelines," IEEE Trans. Electromagn. Compat., vol. 59, no. 5, pp. 1424-1432, Oct. 2017, doi: 10.1109/TEMC.2017.2677199.

[26] Q. Wu, H. Bruns, and C. Schuster, "Characteristic mode analysis of radiating structures in digital systems," IEEE Electromagn. Compat. Mag., vol. 5, no. 4, pp. 56-63, Fourth 2016, doi: 10.1109/MEMC.2016.7866235.

[27] X. Wang et al., "Investigation of the radiation mechanism for highspeed connectors," in 2017 IEEE 26th Conference on Electrical Performance of Electronic Packaging and Systems (EPEPS), Oct. 2017, pp. 1-3, doi: 10.1109/EPEPS.2017.8329711.

[28] X. Yang et al., "EMI Radiation Mitigation for Heatsinks Using Characteristic Mode Analysis," in 2018 IEEE Symposium on Electromagnetic Compatibility, Signal Integrity and Power Integrity (EMC, SI PI), Jul. 2018, pp. 374-378, doi: 10.1109/EMCSI.2018.8495291.
[29] Y. S. Cao, M. Ouyanz, Y. Wang, and J. Fan, "EMI Modeling for Antenna-Chassis System Using Characteristic Mode Analysis," in 2018 IEEE Symposium on Electromagnetic Compatibility, Signal Integrity and Power Integrity (EMC, SI PI), Jul. 2018, pp. 181-186, doi: 10.1109/EMCSI.2018.8495389.

[30] J. Hunter, Y. Liu, D. Floyd, A. Hassan, V. Khilkevich, and D. Beetner, "Characterization of the Electromagnetic Coupling to UAVs," presented at the Annual Directed Energy Science and Technology Symposium, Mar. 2018.

[31] M. Leone, "Radiated susceptibility on the printed-circuit-board level: simulation and measurement," IEEE Trans. Electromagn. Compat., vol. 47, no. 3, pp. 471-478, Aug. 2005, doi: 10.1109/TEMC.2005.850682.

[32] S. Xing, S. Li, W. Hong, and X. Liu, "Using GTEM Cell to Measure RCS of Electrically Small Scatterers," IEEE Antennas Wirel. Propag. Lett., vol. 10, pp. 596-598, 2011, doi: 10.1109/LAWP.2011.2158795.

[33] S. O. Land, R. Perdriau, and M. Ramdani, "Taking Into Account GTEM Field Nonuniformities in Radiated Immunity Simulations Through a Simple Measurement," IEEE Trans. Electromagn. Compat., vol. 59, no. 6, pp. 2042-2045, Dec. 2017, doi: 10.1109/TEMC.2017.2691800.

[34] R. Vogt-Ardatjew and F. Leferink, "Experimental plane wave and random field coupling to uniform and non-uniform transmission lines," in 2015 IEEE International Symposium on Electromagnetic Compatibility (EMC), Aug. 2015, pp. 767-772, doi: 10.1109/ISEMC.2015.7256260.

[35] "Simulation for Connectivity, Compatibility, and Radar | Altair Feko." https://www.altair.com/feko/ (accessed Jan. 25, 2021).

[36] S. Huang, J. Pan, C. Wang, Y. Luo, and D. Yang, "Unified Implementation and Cross-Validation of the Integral Equation-Based Formulations for the Characteristic Modes of Dielectric Bodies," IEEE Access, vol. 8, pp. 5655-5666, 2020, doi: 10.1109/ACCESS.2019.2963278.

[37] Y. Chang and R. Harrington, "A surface formulation for characteristic modes of material bodies," IEEE Trans. Antennas Propag., vol. 25, no. 6, pp. 789-795, Nov. 1977, doi: 10.1109/TAP.1977.1141685.

[38] R. Garbacz and E. Newman, "Characteristic modes of a symmetric wire cross," IEEE Trans. Antennas Propag., vol. 28, no. 5, pp. 712-715, Sep. 1980, doi: 10.1109/TAP.1980.1142388.

[39] N. Peitzmeier and D. Manteuffel, "Upper Bounds and Design Guidelines for Realizing Uncorrelated Ports on Multimode Antennas Based on Symmetry Analysis of Characteristic Modes," IEEE Trans. Antennas Propag., vol. 67, no. 6, pp. 3902-3914, Jun. 2019, doi: 10.1109/TAP.2019.2905718. 